\title{
Le vertige intertextuel. Une lecture de Kamel Daoud, Meursault, contre-enquête
}

The intertextual vertigo. A reading of Meursault, contre-enquête by Kamel Daoud

\section{Veronic Algeri}

\section{(2) OpenEdition}

\section{Journals}

Édition électronique

URL : https://journals.openedition.org/rief/4512

DOI : $10.4000 /$ rief. 4512

ISSN : $2240-7456$

Éditeur

Seminario di filologia francese

\section{Référence électronique}

Veronic Algeri, « Le vertige intertextuel. Une lecture de Kamel Daoud, Meursault, contre-enquête », Revue italienne d'études françaises [En ligne], 9 | 2019, mis en ligne le 15 novembre 2019, consulté le 07 décembre 2022. URL : http://journals.openedition.org/rief/4512 ; DOI : https://doi.org/10.4000/rief. 4512

Ce document a été généré automatiquement le 29 septembre 2020.

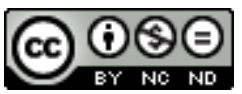

Creative Commons - Attribution - Pas d'Utilisation Commerciale - Pas de Modification 4.0 International - CC BY-NC-ND 4.0

https://creativecommons.org/licenses/by-nc-nd/4.0/ 


\section{Le vertige intertextuel. Une lecture de Kamel Daoud, Meursault, contre- enquête}

The intertextual vertigo. A reading of Meursault, contre-enquête by Kamel Daoud

Veronic Algeri

\section{Introduction}

1 Quand paraît le roman de Kamel Daoud, Meursault, contre-enquête une première fois aux éditions Barzakh, en Algérie, en 2013, et ensuite chez Actes Sud, en 2014, c'est un hommage à Albert Camus que l'on reconnaît dans un jeu intertextuel qui réécrit et corrige, soixante-dix ans plus tard, la trame de L'Étranger. Là où s'inscrit l'injustice d'un crime et est sanctionné l'« effacement de l'indigène »" Daoud souhaite " que justice soit faite $»^{2}$ : désormais l'Arabe assassiné en 1942 sur la plage d'Alger par Meursault a un nom, il s'appelle Moussa Ould el-Assasse, et une histoire que son frère Haroun raconte.

2 Meursault, contre-enquête se donne à lire telle une variation, une réappropriation, une réparation, une inversion ou une suite du texte fondateur, dit-on; chez les lecteurs apparaissent le soupçon d'un paradoxe pour certains entre l'hommage et le mépris, l'hypothèse d'un contrepoint pour d'autres ${ }^{3}$ construit autour d'un équilibre finement travaillé entre les deux éléments d'un binôme qui nous conduit au cœur de l'Histoire ${ }^{4}$. Une histoire toute particulière néanmoins car le colonisateur et le colonisé sont le bourreau et la victime, le maitre et l'esclave, et se présentent comme les deux termes d'une dichotomie subordonnée à un enjeu d'ordre à la fois politique, culturel et psychologique, qui concerne la conscience de soi : en d'autres mots, c'est l'histoire d'une identité qui, au lieu de surgir de la reconnaissance de l'Autre, implique sa mort ${ }^{5}$. En effet, le roman de Daoud se situe, autant que son roman matrice, à l'intérieur de l'histoire coloniale par un crime: l'Arabe qui prépare l'assassinat d'un Français, 
rappelle le meurtre de l'Arabe sans nom dans L'Étranger d'Albert Camus, vingt ans plus tôt.

Quel type de relation se tisse entre ces deux épisodes? « [...] je crois que je voudrais que justice soit faite. Cela peut paraître ridicule à mon âge... mais je te jure que c'est vrai. J'entends par là, non la justice des tribunaux, mais celle des équilibres ${ }^{6}$.

4 En interprétant cette déclaration du narrateur, c'est une sorte de symétrie, au premier abord, qui semble se mettre en place entre ces deux événements mais, sous l'emprise de l'absurde, le dialogue avec le personnage camusien finit rapidement par se refermer sur un monologue, dans le roman de Daoud. La toile de renvois intertextuels se défait et glisse vers une polyphonie perturbée à l'intérieur de laquelle les repères de l'opposition propres de l'imaginaire postcolonial se décomposent, pour laisser surgir un sujet qui prend la parole dans un ensemble de références désormais privées de sens. Le vertige devient la métaphore de la présence de l'homme dans l'Histoire: un nouveau paradigme de l'étrangeté s'installe ainsi au sein de notre roman post-postcolonial et globalisé, qui, en réactualisant l'univers camusien, lit et réagit à la violence des affrontements binaires et idéologisés.

5 À partir de cette hypothèse, et afin d'interroger ses «équilibres ", nous proposons de commencer par détecter les manifestations et les variations de l'intertextualité dans Meursault, contre-enquête. En analysant l'ensemble des relations entre ces deux ouvrages, l'idée est d'abord celle de signaler, au niveau de la structure de notre roman, une reprise ponctuelle d'éléments dans un équilibre d'oppositions symétriques. Puis, dans un deuxième temps, il s'agira de découvrir que le rapport à l'avant-texte renvoie non seulement à des segments de textes autres, mais à un « univers discursif $»^{7}$, un système, autrement dit, au sein duquel se situent les questions de l'intellectuel face à la rhétorique coloniale et les réponses d'un certain humanisme philosophique.

\section{L'hommage}

6 La presse spécialisée, qui a réagi la première à la parution du roman de Daoud, reconnaît d'abord un hommage à Albert Camus, dans la reconstruction minutieuse d'un ensemble de références que l'auteur souhaite signaler à un lecteur avisé, comme dans une sorte de mimétisme stylistique et d'adhérence intellectuelle. Ce roman paraît une première fois sur demande de la maison d'édition algérienne, pour célébrer Camus à l'occasion du centenaire de sa naissance et son chef-d'œuvre, L'Étranger, déjà considéré comme un classique par ses contemporains. Jean-Paul Sartre le célèbre pour :

[...] le tour de ses raisonnements, la clarté de ses idées, la coupe de son style d'essayiste, et un certain genre de sinistre solaire, ordonné, cérémonieux et désolé, tout annonce un classique, un méditerranéen. [...] Il n'est pas jusqu'à sa méthode qui ne fasse penser aux anciennes "géométries passionnées" de Pascal, de Rousseau $\left[\ldots . .{ }^{8}{ }^{8}\right.$

Daoud lui adresse la même reconnaissance : « Il écrit si bien que ses mots paraissent des pierres taillées par l'exactitude même »; «[...] son monde est propre, ciselé par la clarté matinale, précis, net, tracé à coups d'arômes et d'horizons " ${ }^{9}$. La reprise et transformation de l'œuvre de Camus, semble surgir d'une image à l'intérieur de laquelle se déploie une sorte de solidarité entre les techniques narratives, la morale et une certaine métaphysique. 
8 L'intertextualité est, dans un certain nombre de cas, explicite au niveau du péritexte : «L'auteur a cité parfois en les adaptant, certains passages de L'Étranger d'Albert Camus (éd. Gallimard, 1942). Le lecteur les retrouvera en italiques $»^{10}$, peut-on lire dans l'édition française.

9 Ailleurs, ce dispositif se manifeste à travers un ensemble de renvois qui se détachent de la structure diégétique comme des îlots intertextuels. L'auteur introduit en abîme des passages entiers de L'Étranger qu'il prend le soin de mettre entre guillemets: «"La maison était adossée à des rochers et les pilotis qui la soutenaient sur le devant baignaient déjà dans l'eau" " "1 $^{11}$ "...tout était ombre et chaque objet, chaque angle, toutes les courbes se dessinaient avec une confusion insultante pour la raison" $»^{12}$.

Parfois, ce lien est même thématisé et L'Étranger devient :

[...] le livre. Il avait un format assez petit. Une aquarelle était reproduite sur la couverture, représentant un homme en costume, les mains dans les poches, tournant à moitié le dos à la mer, située à l'arrière-plan. Des couleurs pâles, des pastels indécis. C'est ce dont je me souviens. Le titre en était L'Autre, le nom de l'assassin était écrit en lettres noires et strictes, en haut à droite : Meursault. ${ }^{13}$

11 La référence au romancier du XXe siècle ne se borne pas à ce roman, et l'intertextualité, explicite par rapport à l'œuvre palimpseste, s'établit de façon implicite avec La Chute ${ }^{14}$. Haroun est dans un café d'Oran et s'adresse à un « inspecteur universitaire $»^{15}$, comme un demi-siècle plus tôt Jean-Baptiste Clamence se confessait à un homme sans voix, dans un bar d'Amsterdam. Ce lieu de la proximité physique, du dialogue désinvolte et de la consommation d'alcool, est un topos largement exploité par l'écriture littéraire, et devient ici le symbole d'une liberté menacée par le fondamentalisme religieux. Contre ces corps qui se cachent et cette voix qui se tait, Daoud s'insurge aussi bien dans ses romans $^{16}{ }^{\text {que dans ses }}$ Chroniques $^{17}$, comme si dans «le territoire d'une langue inconnue

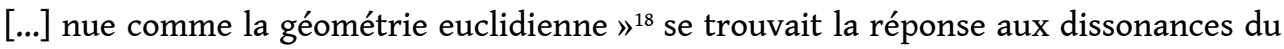
monde. Cette focalisation particulière participe à l'intensité dramatique qui entoure le narrateur omniscient, enfermé dans un point de vue unique, face à un interlocuteur rendu muet par une prise de la parole enragée, dans l'urgence de l'actualité.

De tels motifs interpellent l'œuvre de Camus et installent le roman de Daoud dans une filiation voulue et travaillée.

\section{Opposés dans l'Histoire}

13 L'ensemble des relations intertextuelles renversent le point de vue, inversent le lien de cause à effet et transposent les indications temporelles et spatiales dans une autre dimension, comme à l'intérieur d'un jeu de miroirs.

14 C'est le cas de l'incipit de notre roman, «Aujourd'hui, M'ma est encore vivante " ${ }^{19}$, qui évoque celui de L'Étranger. La place du narrateur est désormais occupée par Haroun, Meursault n'est plus qu'un roumi et Marie devient Meriem. Les personnages évoluent sur le fond d'une lumière et d'une atmosphère générale bouleversées : le soleil de 14 heures qui précise l'heure du crime est remplacé par la lune de deux heures du matin; l'éclat des lumières de la morgue s'oppose aux pièces sombres de la maison qui semblent abriter une veillée funèbre.

15 Ce dispositif performatif concerne aussi le quotidien, dans la parfaite correspondance des codes sociaux et religieux des deux mondes auxquels appartiennent le colon et 
l'indigène: Meursault s'ennuie le dimanche alors qu'Haroun s'ennuie le vendredi; le prêtre est désormais l'imam.

Tous les éléments narratifs participent à la mise en place d'un équilibre qui lie les deux ouvrages et les oppose dans l'Histoire : si L'Étranger propose une " version [...] injuste $»^{20}$ encourageant le silence et l'oubli, la contre-enquête de Daoud défend la « justice [...] des équilibres » à travers un projet qui consiste à retrouver l'origine du crime.

Dans l'idée de composer un monde contre le monde sans camusien, nous observons que le texte de Daoud s'ouvre alors à d'autres intertextualités qui rendent encore plus évident le renversement du point de vue. Afin de reconstruire le déroulement des événements qui, dans l'Étranger, s'enchaînent par "un lien causal sous la pure apparence de la succession $"^{21}$, comme une séquence d'instants, "sans lendemain $»^{22}$, le narrateur oppose à la routine une chronologie, au deuil éternel de la mère et à l'immensité du crime, « une vengeance $»^{23}$. Contre le meurtre insensé de l'Arabe sans nom, Haroun s'insurge alors afin que l'affaire soit jugée, souhaitant qu'un procès soit instruit avec un témoin, un inspecteur et les nombreuses occurrences de termes qui s'approchent du champ sémantique du droit.

Contre Meursault qui, comme Sisyphe ${ }^{24}$, vit dans le présent d'un éternel recommencement, se dresse Haroun, que le hasard a fait naufrager dans un environnement auquel il n'appartient pas et qui, tel le Robinson Crusoé de Daniel Defoe, souhaite réagir à son égarement, en se réappropriant ses nouvelles références : « Tu sais, ici à Oran, ils sont obsédés par les origines. [...] Tout le monde veut être le fils unique de cette ville, le premier, le dernier, le plus ancien. Il y a de l'angoisse de bâtard dans cette histoire, non? $»^{25}$.

Au moyen de cette opération d'appropriation, la citation «parle autrement le discours de l'autre » et permet moins à l'auteur de « prendre possession d'autrui que de soi ${ }^{26}$.

Si «l'homme absurde n'explique pas, il décrit »" la quête identitaire, au contraire, informe toute la structure énonciative du roman et motive à la fois l'enquête judiciaire et l'écriture littéraire au moyen d'une langue capable de «nommer autrement les choses et d'ordonner le monde $»^{28}$.

21 En effet, le narrateur envisage un récit-origine en renversant celui qu'il semble désigner comme le point de vue du discours monolithique occidental : c'est la réponse à l'oppression coloniale et au " phonocentrisme » du droit si l'on suit la thèse de Gayatri Spivak $^{29}$; c'est la théâtralisation d'un espace binaire au sein duquel se produit le conflit de la colonisation et sa revanche réparatrice, dans la métaphore spatiale produite par la théorie de Jean-Marc Moura ${ }^{30}$; c'est l'Orient qui s'oppose à l'Occident dans un renversement de perspectives qu'Edward Saïd reconduit à la stratégie du rewriting, consistant à réécrire la grande Histoire et réinterpréter son parcours individuel au moyen d'une narration rivale, empruntant à l'héritage littéraire occidental pour lui imposer une mutation au contact d'une autre culture et d'une autre esthétique, contre le point de vue historique colonial ${ }^{31}$. Comme le rappelle François Cusset, le programme des subaltern studies, au prisme desquels cette intertextualité peut s'expliquer, permet "[...] aux théoriciens postcoloniaux d'extraire le récit du colonisé de la trame historique dominante, ce "mythe" occidental, pour en faire le point de départ d'une autre pensée de l'histoire, d'une contre-histoire $»^{32}$.

Moussa est l'écho de Meursault, le roman de Daoud, algérien, répond au roman de Camus, français, il représente son double: il nomme l'Arabe, le fait sortir de son 
anonymat, lui rend justice, car le discours colonial a privé l'indigène non seulement de son nom, mais aussi de sa géographie et de son histoire ${ }^{33}$.

Les relations explicites et implicites que nous avons décodées sur la base de cette opposition constitutive, produites par le jeu de l'intertextualité, mènent à l'interprétation d'un système de références qui dépassent les limites du texte pour atteindre l'espace et le temps.

Dans le projet littéraire de Daoud, l'opposition concerne notamment les dates de l'histoire coloniale : le 5 juillet 1942 Meursault tire sur l'Arabe et le 5 juillet 1962 le roumi Joseph Larquais est assassiné par Haroun.

Plus encore, cette symétrie engage les éléments biographiques de nos deux auteurs, leur place et leur position par rapport à cette même Histoire : Camus est journaliste à Alger et Daoud est journaliste à Oran. Or il se trouve que ces deux villes se conforment, elles aussi, à une sorte de disposition bipolaire car la prise d'Alger, qui a lieu en 1830, marque le début de la colonisation, et à Oran, en 1962, a lieu le massacre de civils européens après la reconnaissance officielle de l'Indépendance.

La critique est d'accord et propose ce constat en guise de conclusion : "En revisitant Camus et son Étranger, [on interroge] deux trilogies, celle qui hante l'âme tourmentée des Algériens - Camus-Meursault-l'Arabe - et l'autre qui hante la conscience troublée

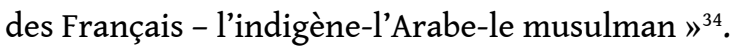

\section{Ni victimes ni bourreaux}

Pour Daoud, écrire avec Camus équivaut à une opération plus ample que celle qui met en contact deux textes. Cela veut dire écrire avec l'histoire coloniale, son discours et sa plaie toujours à vif, comme cette mère qui « Aujourd'hui est encore vivante ».

L'exhibition du rapport à l'hypotexte camusien signale en effet une complexification que nous allons tenter d'éclairer. Au sein d'une écriture «à tiroirs et haletante ${ }^{35}$, la voix qui appartient d'abord à Haroun, finit par prendre la place de celle de Meursault : "Mais souvent aussi je retombe, je me mets à errer sur la plage, pistolet au poing, en quête du premier Arabe qui me ressemble pour le tuer ${ }^{36}$.

Les dispositifs de théâtralisation d'un affrontement binaire s'épuisent rapidement ${ }^{37}$ : la voix du narrateur se superpose à celles de ses interlocuteurs dans un mouvement de concentration polyphonique, en même temps que les parcours de vie des personnages déçoivent l'horizon d'attente du lecteur. Nous apprenons par exemple que Haroun, scolarisé dans les années 1950 à Hadjout, anciennement Marengo, a déserté la cause révolutionnaire et qu'il ne sera pas poursuivi pour le meurtre de Joseph Larquais, «[...] mais parce qu'il n'a pas tué de Français au bon moment c'est-à-dire pendant le temps de la guerre pour l'indépendance de l'Algérie ${ }^{38}$.

S'intensifiant au fil des pages, ces nouvelles informations décloisonnent la morphologie d'un système idéologique qui, des deux côtés, entretient la haine de l'Autre.

31 Une question semble surgir alors de cette structure énonciative à la "mécanique ondulatoire $»^{39}$, concernant la place des uns et des autres face à la colonisation.

Dans ce même "univers farouche et limité de l'homme $»^{40}$, Camus a dû chercher ses réponses. Le quotidien de Meursault est enregistré par une séquence parataxique de gestes et de phrases juxtaposées, sans rapport de coordination ni de subordination; le 
choix du temps verbal, ce passé composé longuement analysé, par Sartre notamment, participe à la mise en place de "la solitude de chaque unité phrastique $»^{41}$ comme à l'intérieur d'un monologue où l'abondance de locutions temporelles place le locuteur en dehors de toute chronologie ${ }^{42}$. Sans liens signifiants entre les faits, une "cloison vitrée $»^{43}$, «transparente aux choses et opaque aux significations $»^{44}$, laisse apercevoir un monde dirigé par le déterminisme d'une "économie mécanique " ${ }^{45}$, et l'on finit par croire que Meursault a tiré sur l'Arabe à cause d'une insolation, le regard dans le vide, la conscience rayée.

S'il développe une sorte de neutralité morale, Meursault refuse aussi le caractère déraisonnable de l'Histoire. Le héros de Camus est lucide, innocent et idiot: « Il est étranger à ce monde et il fait de l'absurde non pas ce qui dérange et brise tout, mais ce qui est susceptible d'arrangement et ce qui même arrange tout $»^{46}$. L'homme trouve alors un sens dans la conscience de la répétition régulière de l'effort sans fin de vouloir refaire le monde en dehors de l'Histoire.

La perspective sociocritique postcoloniale semble être dépassée par une réflexion métaphysique.

À côté de Meursault, se dresse alors Haroun qui, sur la tombe vide de son frère, devient aussi un personnage sisyphéen : "C'est dans cet endroit que je me suis éveillé à la vie, crois-moi. C'est là que j'ai pris conscience que j'avais droit au feu de ma présence au monde [...] malgré l'absurdité de ma condition qui consistait à pousser un cadavre vers le sommet du mont avant qu'il ne dégringole à nouveau, et cela sans fin $»^{47}$.

Daoud et Camus, autant que les narrateurs de leurs romans, ne sont pas opposés dans l'Histoire car tous deux brouillent les clivages d'une opposition érigée d'abord par le système colonial puis, à partir de l'Indépendance, entretenue par la rhétorique du discours national. Ce schéma d'opposition bipolaire, nécessaire comme l'est un marqueur identitaire et absurde comme l'est tout régime racial basé sur la haine de l'autre, continue de s'imposer aux héritiers de l'histoire coloniale, tel un appareil de déshumanisation, comme l'entend Sartre ${ }^{48}$, ou un système d'aliénation, comme dans l'analyse de Saïd qui écrit à ce propos que : «the colonized people [...] had freed themselves [...] but remained victims of their past $»^{49}$.

Né en 1970 et faisant partie de la génération de la post-indépendance ${ }^{50}$, Daoud considère que le drame du peuple algérien consiste à continuer à entretenir une sorte de "nostalgie du bilatéral pur et dur ${ }^{51}$ dans la représentation de soi et la relation à l'Autre. En 1962, le colonisé se retrouve face à lui-même pour la première fois depuis toujours, il est uni à ses frères par un drapeau et une langue qui ne sont pas les siens, il est condamné à la mystification de la guerre de libération, et finit par trouver sa liberté dans le paradoxe de l'adoption de la langue du colonisateur.

Butin de guerre pour l'écrivain algérien Kateb Yacine, langue de l'Autre mais aussi autre langue, langage poétique, pour Assia Djebar ${ }^{52}$, la langue française représente un espace d'affranchissement. Dans cette acception bien particulière, Daoud énonce sa propre politique de la langue : faire « des mots du meurtrier et de ses expressions [un ] bien vacant $"^{53}$, sans propriétaire (le colon l'aurait-elle abandonnée ou perdue) ou sans possesseur (l'indigène l'aurait-elle volée), pour sublimer le réel, comme l'a fait Camus, qui « savait raconter, au point qu'il a réussi à faire oublier son crime $»^{54}$, " pour parler à la place du mort, continuer un peu ses phrases $»^{55}$. 
La contre-enquête ne peut aboutir. Reste Sisyphe et son mythe qui inspire la révolte: jour après jour, le chroniqueur lit l'actualité et raconte d'autres histoires possibles audelà du trouble que les violences de la décennie noire ont projeté sur les références identitaires des peuples dans la globalisation, encore et toujours colonisé : « Quel drôle de métier. D'ailleurs est-ce réellement un métier? S'amuser à refaire le monde chaque matin, le porter comme Atlas, comme Sisyphe... dans un recommencement sans fin?» 56 .

La langue que Daoud s'approprie est littéraire dans l'écriture romanesque mais aussi dans ses témoignages journalistiques. Tel un roman, la chronique est une écriture de la jouissance et du jeu (non pas de la justice mais de la justesse), où la langue française, capable d'« éclairer les plis du quotidien étouffant entre le minaret et la caserne ${ }^{57}$, n'appartient ni aux victimes ni aux bourreaux.

41 Ce binôme, en dehors duquel Daoud semble vouloir se situer à travers le recours à la pratique de l'écriture, est aussi refusé par Camus.

Dans la série d'articles intitulée Ni victimes ni bourreaux, il écrit: " on nous demande d'aimer ou de détester tel ou tel pays et tel ou tel peuple. Mais nous sommes quelquesuns à trop bien sentir nos ressemblances avec tous les hommes pour accepter ce choix " 58 .

\section{Le vertige}

Une fois repoussées toutes politiques identitaires issues d'une éthique de l'antagonisme, celles du discours colonial aussi bien que celles du récit-origine de la nation algérienne, que peut l'intellectuel?

Désormais, la question à laquelle Daoud semble vouloir répondre n'est plus de connaître la place des uns et des autres face à la colonisation, mais plutôt de savoir resituer l'individu dans l'Histoire. À notre tour, nous souhaitons y parvenir en suivant notamment les très nombreuses occurrences du mot « vertige » dans Meursault, contreenquête.

45 «[...] juste après l'Indépendance, je suis revenu à Alger, résolu à mener ma propre enquête. Mais penaud j'ai fait demi-tour à la gare. Il faisait chaud, je me sentais ridicule dans mon costume de ville et tout allait trop vite, comme un vertige $[. ..] »^{59}$. La tentative d'échapper à la routine d'un ordre causal et d'établir un "enchaînement chronologique " ${ }^{60}$, échoue, car la vengeance n'est pas réparatrice et dans le crime il n'y a pas de libération. Une fois l'équilibre perdu, le vertige s'empare de l'homme : «J'avais tué et cela me donnait un vertige incroyable $»^{61}$.

6 Ce sentiment donne l'illusion que son corps ou que les objets environnants sont animés d'un mouvement d'oscillation, mais décrit aussi l'attraction irrésistible d'une chute éprouvée au-dessus du vide, ou d'une date. Car dans notre roman, l'Histoire et ses hommes se donnent rendez-vous le 5 juillet. Si en 1942 et en 1962 sont assassinés respectivement l'Arabe et le roumi, l'équilibre à peu près parfait qui aurait assuré « que justice soit faite ${ }^{62}$, se défait lorsqu'on découvre que l'assassinat est commis deux jours après la reconnaissance officielle de l'Indépendance, et que la date de sa proclamation, que le général De Gaulle doit faire le soir du 5 juillet, arrive le jour du $132^{\mathrm{e}}$ anniversaire de la prise d'Alger par les Français. 

proche d'un jeu de correspondances ludiques, qui tente en vain de donner un sens au hasard. Le narrateur l'explique ainsi : «Je pouvais passer de vie à trépas et de l'au-delà au soleil en changeant seulement de prénom: moi Haroun, Moussa, Meursault ou Joseph. La mort, aux premiers jours de l'Indépendance, était aussi gratuite, absurde et inattendue qu'elle l'avait été sur une plage ensoleillée de $1942 »^{63}$. Les crimes, à travers lesquels l'Histoire se reproduit, dans l'élan vain d'une aspiration à un idéal par exemple, s'enchainent dans le vertige d'une répétition injuste où l'individu est condamné à se perdre. Comme Haroun qui avoue : «J'ai connu les vertiges de l'homme qui possède un secret bouleversant et je me suis promené ainsi, avec une sorte de monologue sans fin dans ma tête ${ }^{64}$.

Cioran, que l'auteur cite en exergue à son roman, l'exprime ainsi : "L'heure du crime ne sonne pas en même temps pour tous les peuples. Ainsi s'explique la permanence de l'histoire " ${ }^{65}$. Dans cet inexorable flux d'événements, Daoud renonce à rendre aux victimes la $"$ justice absurde ${ }^{66}$ des tribunaux, mais compose la justesse de l'écriture littéraire, comme si une fois déçues les attentes d'un ordre moral, il ne restait plus que les formes d'un virtuosisme esthétique. On se demande alors si Meursault a tué par le déterminisme ou par le hasard de l'histoire et si l'Arabe avait « un révolver, une philosophie ou une insolation? $»^{67}$. Sans victimes et sans bourreaux, sans hiérarchie et sans références, Haroun tombe dans le vide anéantissant d'une histoire qu'il ne peut que rejouer à l'infini : «J'éprouve juste une sorte de lassitude, l'envie de dormir souvent et, parfois, un immense vertige $»^{68}$. Mais le vertige correspond aussi à la métaphore d'un sentiment d'étrangeté, comme le «trouble mental dans lequel le malade perd le sentiment de sa réalité personnelle, reconnaît mal le monde environnant $»^{69}$.

51 Cette fracture coloniale continue de situer les anciens colonisateurs et les nouveaux Algériens à l'intérieur d'une opposition qui concerne aussi le rapport entre les littératures francophones et la littérature de l'Hexagone, dans les termes d'une hiérarchisation entre une périphérie et un centre. Un rapport que l'ouvrage de Daoud a déstabilisé, par une démarche transgressive, et cela au moins pour deux raisons: d'abord un écrivain algérien reprend un auteur classique de la littérature française, puis son roman paraît en Algérie, à la périphérie du marché éditorial, avant d'être publié en France, au sein des institutions de la sacralisation littéraire ${ }^{70}$. S'agit-il, comme le soupçonne Sylvie Ducas, d'« une extrême ambition qui ne s'avoue pas, celle d'un écrivain algérien rêvant de devenir un grand "écrivain français" »"11 ? Ou bien, est-ce la permanence d'un rapport de force qui lie, dans le conflit et l'opposition, par une sorte d'attirance, le colonisé au colonisateur ? Cette force mimétique est l'objet de l'analyse d'Albert Memmi.

52 Le colon attire l'indigène pour mieux le repousser : « [...] c'est sa langue maternelle (du colon) qui permet les communications sociales; même son costume, son accent, ses manières finissent par s'imposer à l'imitation du colonisé $[. ..] »^{72}$.

53 La perversion de la relation coloniale se manifeste alors dans sa physionomie qui ressemblerait à une pyramide dans laquelle l'indigène souhaite prendre les distances du musulman et s'identifier au Français, et le colonisé finit par être le colonisateur :

[...] dans un grand élan qui m'emportait vers l'occident, qui me paraissait le parangon de toute civilisation et de toute culture véritables, j'ai d'abord tourné allègrement le dos à l'Orient, choisi irrévocablement la langue française, me suis 
habillé à l'italienne et ai adopté avec délices jusqu'aux tics des Européens. En quoi d'ailleurs, j'essayais de réaliser l'une des ambitions de tout colonisé, avant qu'il ne passe à la révolte. ${ }^{73}$ l'analyse de l'actualité dans laquelle surgit une réflexion sur le rapport de l'intellectuel à la politique et, face à un régime de discours qui continue d'être hanté par la fracture coloniale, une forme de liberté est revendiquée. L'Indépendance perd sa lettre majuscule et se décline au pluriel, à travers la référence à l'auteur des Chroniques algériennes ${ }^{76}$ : son combat pour la vérité en dehors des appartenances idéologiques et des partis politiques ; son attachement à l'Algérie qui le fait sentir en exil en France ; la dénonciation lucide des injustices du système colonial; la revendication d'une issue dans l'égalité sont les thèmes inspirateurs d'un combat dont Daoud revendique à plusieurs reprises l'ascendance. complexité et Kamel Daoud, qui semblait s'insérer dans la lignée « contrapuntique » de Saïd ${ }^{77}$ par ce biais littéraire, est encore l'homme révolté, faisant l'expérience de l'absurde.

«Coincé[s] entre deux histoires $»^{78}$ et victimes de leurs rhétoriques aliénantes, se situant d'une certaine manière à un bout et à l'autre de cette fracture qui fait parader sur les deux fronts de la Méditerranée le nous et le eux, les victimes et les bourreaux, ouvrant un abîme au cœur d'une identité absurde pour Camus, peut-être vertigineuse pour Daoud, nos deux auteurs semblent réaliser que l'Autre, loin de servir à alimenter une haine paralysante, permet de 4 creuser les perspectives de sa solitude $»^{79}$, que la mesure de l'homme n'est pas dans l'opposition à son frère mais plutôt dans l'immensité comme contrepoint de sa petitesse.

\section{NOTES}

1. S. Lapaque, «Meursault, contre-enquête de Kamel Daoud: une réécriture de Camus ", dans Le Figaro, 16 octobre 2014, consulté le 15/11/2018, URL: <http://www.lefigaro.fr/livres/ 2014/10/16/03005-20141016ARTFIG00015--meursault-contre-enquete-de-kamel-daoud-unereecriture-de-camus.php>.

2. K. Daoud, Meursault, contre-enquête, Arles, Actes Sud, 2014, p. 16 (dorénavant MCE). 
3. M. Séry, « Kamel Daoud double Camus. Avec "Meursault, contre-enquête”, l'écrivain algérien a réécrit "L'Étranger" - du point de vue arabe. Superbe ", dans Le Monde, 25 juin 2014, consulté le 15/11/2018, URL : <https://www.lemonde.fr/livres/article/2014/06/25/kamel-daoud-doublecamus_4445128_3260.html>.

4. Cette démarche est à la base du roman de K. Daoud, Le Peintre dévorant la femme, Paris, Stock, 2018. L'Orient et l'Occident s'opposent, à travers une série de couples dichotomiques : le sauvage et le civilisé, le corps voilé et le corps nu, Abdellah et Picasso, le djihadiste et l'artiste, le désert et l'idéal de pureté unique pour le premier, le musée et la défense des différents patrimoines de l'humanité pour l'autre.

5. Hegel a déjà pensé le binôme du maître et de l'esclave comme une relation paradoxale basée sur l'indifférence et la dépendance réciproques. G. W. F. Hegel, Indépendance et dépendance de la conscience de soi : domination et servitude, dans Phénoménologie de l'esprit [1807], tr. fr. J. Hyppolite, Paris, Aubier-Montaigne, t. 1, 1978, p. 145 et sq.

6. $M C E$, p. 16.

7. J. Kristeva, «Le mot, le dialogue, le roman », dans Id., Sémeiotiké, Paris, Seuil, 1969, p. 82-112, p. 84.

8. J.-P. Sartre, « Explication de L'Étranger », dans Id., Situations, I, Paris, Gallimard, 1947, p. 99-121, p. 102.

9. MCE, p. 12.

10. Ibid., p. 4.

11. Ibid., p. 77.

12. Ibid., p. 115.

13. Ibid., p. 137.

14. A. Camus, La Chute, Paris, Gallimard, 1956.

15. MCE, p. 30.

16. K. Daoud, Le Peintre dévorant la femme, cit., p. 180.

17. Id., Mes indépendances. Chroniques 2010-2016, Arles, Actes Sud, 2017.

18. MCE, p. 110.

19. Ibid., p. 11.

20. Ibid., p. 29.

21. J.-P. Sartre, art. cit., p. 118.

22. MCE, p. 29.

23. Ibid., p. 48.

24. A. Camus, Le Mythe de Sisyphe. Essai sur l'absurde, Paris, Gallimard, 1942.

25. MCE, p. 21.

26. A. Compagnon, La Seconde Main, ou le travail de la citation, Paris, Seuil, 1979, p. 351 et p. 356.

27. J.-P. Sartre, art. cit., p. 105.

28. MCE, p. 47.

29. G. Spivak, In Other Words: Essays in Cultural Politics, New York, Routledge, 1988, p. 213.

30. J.-M. Moura, Littératures francophones et théorie postcoloniale, Paris, PUF, 1999.

31. E. W. Saïd, Orientalism, New York, Pantheon, 1978.

32. F. Cusset, « Politiques identitaires ", dans Id., French Theory, Paris, La Découverte, p. 143-178, p. 155.

33. E. W. Saïd, « Representing the Colonized: Anthropology's interlocutors », dans Critical Inquiry, 15,1989 , p. 205-225.

34. M. Harzoune, "Kamel Daoud, Meursault, contre-enquête ", dans Hommes et migrations, 1308, 4, 2014, p. 195.

35. F. Tilikete, « Meursault, contre-enquête ou la nécessaire réécriture », dans Africa Review of books/ Revue Africaine des Livres, 11, 2, septembre 2015, p. 19-20, p. 19.

36. MCE, p. 147. 
37. Ch. Bonn, «La littérature francophone serait-elle sortie du face-à-face post-colonial ? ", dans Modern \& Contemporary France, 10, 4, 2002, p. 483-493.

38. E. Caduc, "Postérités d'Albert Camus chez les écrivains algériens de Kateb à Sansal », dans Loxias-Colloques, 4, «Camus: "un temps pour témoigner de vivre" (séminaire)», consulté le 15/11/2018, URL : <http://revel.unice.fr/symposia/actel/index.html?id=686>.

39. M. Harzoune, art. cit., p. 195.

40. A. Camus, Le Mythe de Sisyphe, dans Id., Le Mythe de Sisyphe. Essai sur l'absurde, cit., p. 161-168, p. 167.

41. J.-P. Sartre, art. cit., p. 117.

42. M.-G. Barrier, L'Art du récit dans L'Étranger de Camus, Paris, Nizet, 1962.

43. J.-P. Sartre, art. cit., p. 114.

44. Ibid., p. 115.

45. Ibid., p. 121.

46. M. Blanchot, «De l'Angoisse au langage », dans Id., Faux pas [1943], Paris, Gallimard, 1971, p. $65-71$, p. 70.

47. MCE, p. 57.

48. J.-P. Sartre, «Préface », dans A. Memmi, Portrait du colonisé. Portrait du colonisateur, [1966], Paris, Gallimard, 1985.

49. «[...] les peuples colonisés [...] se sont libérés [...] mais sont toujours victimes de leur passé », E. W. Saïd, art. cit. p. 207 [Nous traduisons].

50. Ch. Chaulet Achour, «Une variation algérienne sur l'écriture camusienne: Meursault, Contreenquête de Kamel, Daoud (2013)", "Albert Camus et l'Algérie ", Lyon, 30 janvier 2014 Association Coup de Soleil en Rhône Alpes, consulté le 15/11/2018 URL: <http:// christianeachour.net/images/data/telechargements/2014/A283.pdf>.

51. MCE, p. 32.

52. V. Algeri, L'Histoire de soi dans la langue de l'autre. La Polyphonie linguistique dans l'œuvre d'Assia Djebar, Roma, Aracne, 2014.

53. MCE, p. 12.

54. Ibid., p. 11.

55. Ibid., p. 12.

56. Ibid., p. 9.

57. K. Daoud, Mes indépendances. Chroniques 2010-2016, cit. p. 16.

58. A. Camus, « Ni victimes ni bourreaux. 19-30 novembre 1946 », dans Id., Camus à Combat, éd. J. Lévi-Valensi, Paris, Gallimard, « Cahiers Albert Camus 8 », 2002, p. 641.

59. MCE, p. 33.

60. J.-P. Sartre, art. cit., p. 121.

61. MCE, p. 121.

62. Ibid., p. 16.

63. Ibid., p. 115.

64. Ibid., p. 148.

65. Ibid., p. 7.

66. J.-P. Sartre, art. cit., p. 111.

67. MCE, p. 14.

68. Ibid., p. 97.

69. P. Janet et F. Raymond, Les Obsessions et la psychasthénie, Paris, Alcan, 1903, p. 42.

70. K. Harchi, Je n'ai qu'une langue et ce n'est pas la mienne, Paris, Pauvert, 2016, p. 27.

71. S. Ducas, "L'entrée en littérature française de Kamel Daoud: "Camus, sinon rien !" ", dans Littératures, 73, 2015, p. 185-197, p. 185.

72. A. Memmi, op. cit., p. 38.

73. Ibid., p. 25. 
74. Ibid., p. 46-47.

75. Ibid., p. 13.

76. A. Camus, Chroniques algériennes 1939-1958, Paris, Gallimard, 2002.

77. E. W. Saïd, "Camus et l'expérience impériale française », dans Id., Culture et Impérialisme [1993], tr. fr. P. Chemla, Paris, Fayard - Le Monde diplomatique, 2000, ch. 7, p. 248-268.

78. MCE, p. 93.

79. Ibid., p. 83.

\section{RÉSUMÉS}

En interrogeant la présence de l'œuvre d'Albert Camus dans le roman Meursault, contre-enquête (Barzakh, 2013 et Actes Sud, 2014) de Kamel Daoud, apparaissent les marques d'un engagement politique et poétique qui surgit aujourd'hui comme hier d'une inquiétude face à l'absence de repères. Deux auteurs, écrivains et journalistes, dialoguent ainsi d'un bout à l'autre de l'histoire coloniale, pour soulever la question fondamentale de la raison de la haine. Daoud avec son Arabe, comme Camus avec son Meursault, trouvent la réponse en dehors des idéologies, dans l'absurde. Est-ce le prix à payer pour se libérer d'un récit hanté par la fracture coloniale?

By questioning the presence of the work of Albert Camus in the novel Meursault, contre-enquête (Barzakh, 2013 and Actes Sud, 2014) by Kamel Daoud, the marks appear of a political and poetic commitment that arises today as yesterday from a concern due to the absence of landmarks.Two authors, both writers and journalists, from one side to the other of colonial history, raise the fundamental question of the reason of hate. Daoud with his Arab, like Camus with his Meursault, find the answer beyond ideologies, but in the absurd. Is this the price to pay to free oneself from a story haunted by the colonial fracture?

\section{INDEX}

Mots-clés : Daoud (Kamel), Camus (Albert), intertextualité, absurde, colonisation

Keywords : Daoud (Kamel), Camus (Albert), intertextuality, absurd, colonization 